\title{
A critical appraisal of phloem-mobile signals involved in tuber induction
}

\author{
Paula Suárez-López* \\ Molecular Genetics Department, Centre for Research in Agricultural Genomics, CSIC - IRTA - UAB - UB, Barcelona, Spain
}

\begin{abstract}
Edited by:
Jurriaan Ton, University of Sheffield,

UK
\end{abstract}

Reviewed by:

Chiou, Tzyy-Jen, Academia Sinica,

Taiwan

Jurriaan Ton, University of Sheffield,

UK

Julia Kehr, University Hamburg,

Germany

David Hannapel, lowa State

University, USA

Tien-Shin Yu, Institute of Plant and

Microbial Biology, Academia Sinica,

Taiwan

\section{*Correspondence:}

Paula Suárez-López, Molecular Genetics Department, Centre for

Research in Agricultural Genomics,

CSIC - IRTA - UAB - UB, Campus UAB,

Bellaterra (Cerdanyola del Vallès),

08193 Barcelona, Spain

e-mail:paula.suarez@cragenomica.es
The identification of FLOWERING LOCUS T (FT) and several FT homologs as phloem-mobile proteins that regulate flowering has sparked the search for additional homologs involved in the long-distance regulation of other developmental processes. Given that flowering and tuber induction share regulatory pathways, the quest for long-distance tuberization signals has been further stimulated. Several tuberization regulators have been proposed as mobile molecules, including the FT family protein StSP6A, the plant growth regulators gibberellins and the microRNA miR172. Although some of these hypotheses are attractive and plausible, evidence that these molecules are transmissible in potato has yet to be obtained. Two mRNAs encoding transcription factors, StBEL5 and POTATO HOMEOBOX 1 (POTH1), are mobile and correlate with tuber induction. However, evidence that StBEL5 or POTH1 are required for tuberization is not available yet. Therefore, there are several good candidates for long-distance molecules in the tuberization process. Further research should test their role as systemic tuberization signals.

Keywords: tuberization, potato, long-distance signaling, FLOWERING LOCUS T, gibberellins
The induction of tuber formation is a key developmental transition for the production of potatoes, one of the most important food crops. Understanding the regulation of tuber induction is essential to devise strategies to improve tuber yield and quality. During the last two decades we have started to comprehend this regulation, with the identification of genes that control tuberization (Jackson, 1999; Abelenda et al., 2011). This has been facilitated by the tremendous progress in understanding the control of flowering, which is similar to tuberization in aspects such as the response to photoperiod and the involvement of phloem-mobile signals (Suárez-López, 2005; Abelenda etal., 2011). This Perspective paper focuses on recent findings that suggest several molecules as candidates for systemic signals controlling tuber induction.

\section{LONG-DISTANCE SIGNALS REGULATE TUBERIZATION AND FLOWERING}

Short day (SD) photoperiods promote tuberization, whereas long days (LDs), high nitrogen levels and high temperatures inhibit or delay tuberization. Within the tuberization process, it is important to distinguish between tuber induction and tuber development and growth. Induction takes place when signals are produced in leaves and transported through the phloem to underground stems (stolons), or when mobile signals that inhibit tuberization are repressed (Jackson, 1999; Suárez-López, 2005). This leads to the initiation of tuber development and growth, which determines tuber shape, number, and weight. Although tuber yield is often used to assess tuber induction, changes in tuber yield can result from alterations in many different factors, including overall plant growth, photoassimilate partitioning, the strength of induction, tuber development, etc. (Ewing and Struik, 1992). The time of tuber initiation is therefore a much better indicator of tuber induction than tuber yield.

Grafting experiments using potato plants induced and noninduced to tuberize demonstrated the existence of transmissible substances decades ago (Gregory, 1956; Chapman, 1958), but the identification of these signals has proven difficult. Recent advances in the study of other developmental processes provide hints for finding long-distance tuberization signals. The intensive search for a phloem-mobile flowering signal, called florigen, has led to the identification of several FLOWERING LOCUS T (FT) family members as leaf-produced proteins that travel to the shoot apical meristem, where they induce flowering (Turck et al., 2008; Tsuji et al., 2013). In Arabidopsis thaliana, FT expression is activated by the transcriptional regulator CONSTANS (CO) in leaf phloem cells in response to floral inductive photoperiods (An et al., 2004; Ayre and Turgeon, 2004).

However, florigen is not a single molecule. Positive and negative transmissible regulators of flowering exist (Bernier, 1988; Matsoukas etal., 2012). Several FT family members can perform these functions. In rice, Heading date $3 \mathrm{a}(\mathrm{Hd} 3 \mathrm{a})$ and RICE FLOWERING LOCUS T 1 (RFT1) act as florigenic signals under different photoperiods (Tamaki et al., 2007; Komiya et al., 2009). In Arabidopsis ATC acts as a mobile repressor or antiflorigen and TWIN SISTER OF FT (TSF) might function as a florigen 
(Yamaguchi et al., 2005; Mathieu etal., 2007; Jang et al., 2009; D'Aloia et al., 2011; Huang et al., 2012). In addition, several FTrelated proteins have been detected in phloem exudates of diverse species (Giavalisco et al., 2006; Lin et al., 2007; Aki et al., 2008).

Many RNAs are present in phloem exudates and a few have been reported to act in long-distance signaling (Sasaki et al., 1998; Ruiz-Medrano et al., 1999; Kim et al., 2001; Doering-Saad et al., 2002; Haywood et al., 2005). Movement of FT and ATC RNAs has been shown, but the FT RNA accelerates flowering less effectively than the protein and the RNA together (Li et al., 2009, 2011; Huang et al., 2012; Lu et al., 2012). Other reports indicate that translocation of the FT protein, but not the RNA, is required to promote flowering (Lifschitz et al., 2006; Mathieu et al., 2007; Notaguchi et al., 2008). These findings suggest that movement of the FT mRNA can help to induce flowering, but movement of the FT protein is much more crucial. In addition to FT proteins and RNA, other types of molecules, such as hormones and metabolites, have been postulated as long-distance floral signals (Turnbull, 2011; Dinant and Suárez-López, 2012).

\section{IS FT A PHLOEM-MOBILE TUBERIZATION SIGNAL?}

Transmissible signals for flowering and tuberization are interchangeable. Tobacco scions induced to flower promote tuberization when grafted onto potato stocks kept under nontuber-inducing conditions (Chailakhyan et al., 1981). When a rice Hd3a-GFP fusion is expressed in potato phloem, it can move across a graft junction to stolons and induce tuber formation (Navarro etal., 2011), suggesting that a similar protein exists in potato. Indeed, several FT-like genes have been identified in this species. One of them encodes StSP3D, which mainly affects flowering, and another encodes StSP6A, which induces tuber formation, a role similar to that of FT in flowering control (Navarro et al., 2011). The effect of StSP6A on tuberization is transmitted through grafts (Navarro et al., 2011). Altogether, these findings strongly suggest that StSP6A is probably a mobile tuberization signal.

There are similarities, but also differences, in the regulation of FT genes. StSP6A is negatively regulated by StCO (Figure 1), a potato $\mathrm{CO}$-like protein that represses tuberization under noninductive LDs (Navarro et al., 2011; González-Schain et al., 2012). StCO does not seem to play a role under SDs (González-Schain et al., 2012). By contrast, Arabidopsis CO promotes FT transcription only under inductive photoperiods (Turck et al., 2008). In rice $H d 3 a$ is repressed or activated by the CO-like protein Hd1 under non-inductive or inductive conditions, respectively, and in addition RFT1 is up-regulated and promotes flowering much later under non-inductive conditions (Tsuji et al., 2013). These differences stress the need to test hypotheses based on flowering-time models, rather than simply extrapolating them to tuberization. Demonstrations that StSP6A moves are therefore eagerly awaited.

Two additional FT family members from potato, StTFL1 and StSP5G, might be related to the tuberization process. StTFL1 mRNA levels are high in stolons before induction and decrease at early stages of tuber development. Overexpression of StTFL1 causes an increase in the number of tubers produced (Guo et al., 2010), suggesting a role in tuber induction or development. The expression pattern of StSP5G suggests that this gene might play an opposite role to that of StSP6A in tuberization control (Navarro et al., 2011; Kloosterman et al., 2013), although a functional analysis of this gene has not been reported so far. Further analyses of StTFL1 and StSP5G to determine their biological functions should be pursued, given that FT-related proteins affect other developmental processes aside from flowering and tuberization (Pin and Nilsson, 2012; Hiraoka et al., 2013). As many FT-like proteins are mobile, it would be worth testing StTFL1 and StSP5G movement.

\section{StBEL5 AND POTH1 mRNAs AS PUTATIVE TRANSMISSIBLE SIGNALS}

Two mRNAs have been proposed as long-distance signals regulating tuberization. StBEL5 and POTATO HOMEOBOX 1 (POTH1) are homeobox transcription factors that interact with each other (Chen et al., 2003). Overexpression of POTH1 increases the number of tubers produced relative to wild-type (WT) plants in in vitro tuberization assays (Rosin et al., 2003). Overexpression of StBEL5 enhances tuber formation under SDs and promotes tuberization under non-inductive LDs. StBEL5 mRNA moves from overexpressing scions to WT stocks and movement correlates with increased tuber yield (Chen et al., 2003; Banerjee et al., 2006). Graft transmission of POTH1 mRNA has also been shown (Mahajan etal., 2012). Transcription of StBEL5 and POTH1 in vascular cells (Banerjee et al., 2006; Mahajan et al., 2012) is consistent with movement of their transcripts through the phloem. Additional experimental approaches support translocation of StBEL5 mRNA and have been previously reviewed (Hannapel, 2010).

However, there are numerous caveats to be aware of when interpreting the movement of StBEL5 and POTH1 RNAs, as well as their effects on tuberization. First, POTH1 has not been shown to affect tuber formation in soil-grown plants. Second, whether StBEL5 and/or POTH1 are required for tuber induction in WT plants has not been demonstrated, as only overexpression alters tuber induction or development. Third, RNA movement has been shown from overexpressing plants, but not from WT plants (Banerjee et al., 2006; Mahajan et al., 2012), and it has not been tested whether movement is required for tuberization. Fourth, POTH1-overexpressing plants exhibit dramatic alterations in the vasculature (Rosin et al., 2003; Mahajan et al., 2012). It is possible that the tuber phenotype of POTH1-overexpressing plants and graft transmission of POTH1 mRNA are indirect consequences of these alterations. Fifth, both POTH1 and StBEL5 are transcribed in stolons, with an increase in StBEL5 transcription at early stages of tuber formation (Banerjee et al., 2006; Mahajan et al., 2012), casting doubts on the need of movement from leaves. Finally, it has not been excluded that movement of StBEL5 and/or POTH1 proteins may occur.

Therefore, although StBEL5 and POTH1 RNAs are able to move, further research is needed to demonstrate whether this has any biological relevance. This can be addressed by simultaneously silencing StBEL5 and POTH1 or several StBEL paralogs, which have been proposed to act redundantly (Chen et al., 2003). Whether the StBEL5 protein moves should also be tested. 


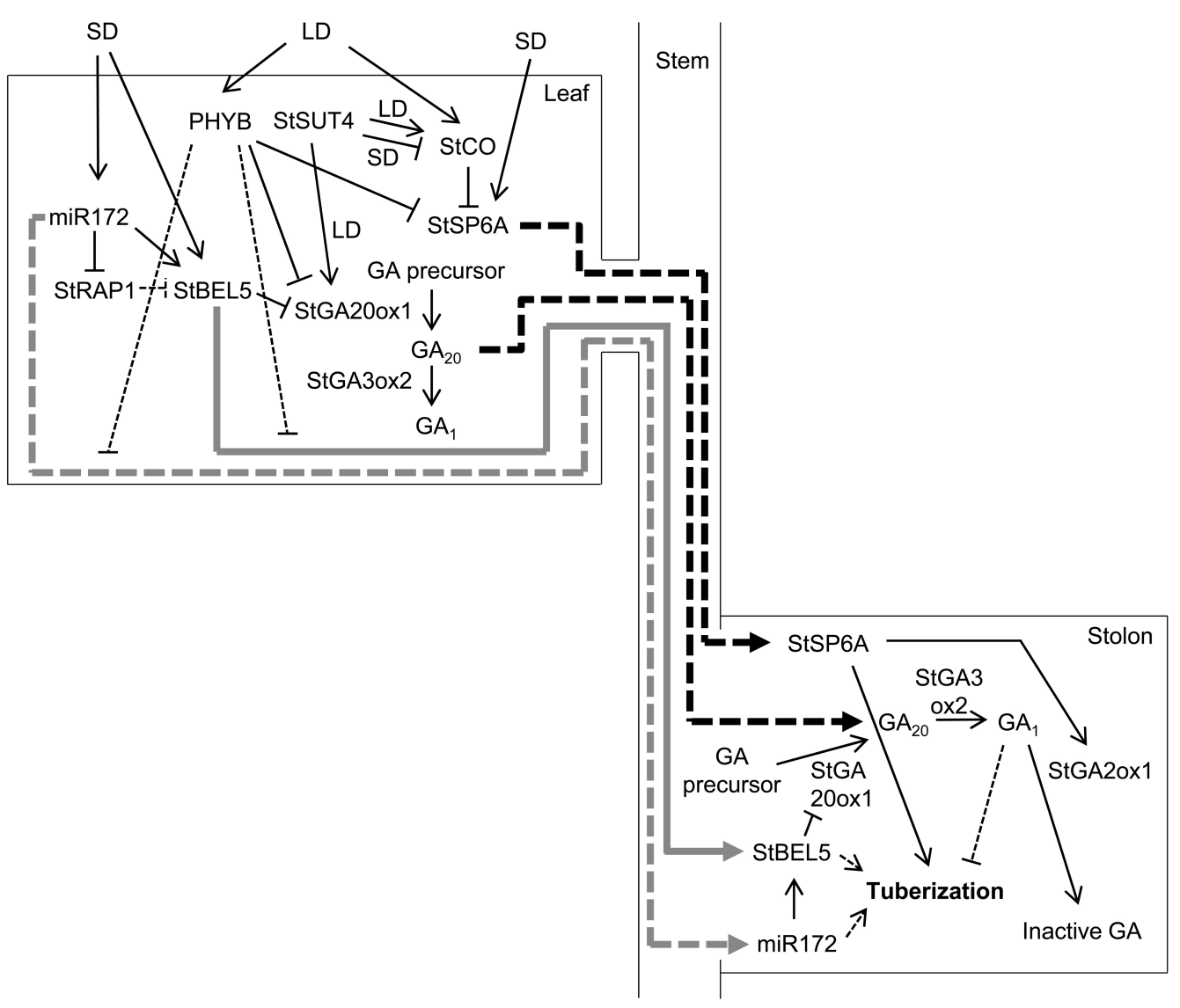

FIGURE 1 | Model for the regulation of tuber induction by phloem-mobile signals. The main candidates for mobile signals are the StSP6A protein, two RNAs - StBEL5 and miR172 - and GAs. The production, and possibly the movement, of these four factors is regulated by a complex genetic network. PHYB, StSUT4, and StCO repress tuberization in response to LDs. GAs also seem to act as repressors, whereas StSP6A and perhaps miR172 and StBEL5 act as tuberization promoters under inductive SD conditions. Under LDs, PHYB represses the expression of StSP6A and StGA200x1, which encodes an enzyme that catalyzes the synthesis of $\mathrm{GA}_{20}$. PHYB up-regulates miR172 and StBEL5 in leaves and down-regulates them in stolons, which might result from a repression of StBEL5 mRNA and miR172 movement from leaves to stolons. Under LDs, StSUT4 induces StGA200x1 and StCO, which represses StSP6A. Under SDs, StSUT4 inhibits StCO, relieving StSP6A repression. In addition to StSP6A, SDs up-regulate miR172 and StBEL5. miR172 induces StBEL5, probably through the repression of
miR172 target genes, such as StRAP1, which would act as StBEL5 inhibitors. StBEL5 represses StGA200x1 in a complex with POTH1. StGA3ox2 catalyzes the conversion of $\mathrm{GA}_{20}$ to $\mathrm{GA}_{1}$, an active GA. StSP6A, StBEL5 mRNA, miR172, and GAs presumably translocate to stolons through the phloem. In the stolons, StSP6A promotes tuber development, at least in part through up-regulation of StGA2ox1, which converts active GAs into inactive forms. miR172 up-regulates StBEL5, which together with POTH1 down-regulates $S t G A 200 \times 1$, reducing the synthesis of active GAs, which repress tuber development. Under LDs, $\mathrm{GA}_{20}$ would move from leaves to stolons and would be converted to $\mathrm{GA}_{1}$, thus repressing tuber development. Under SDs, there would be less $\mathrm{GA}_{20}$ available and tuber development can occur. Thick gray arrows indicate RNA movement, and thick black arrows indicate protein or GA movement. Discontinuous lines indicate that movement or regulation has been suggested, but not demonstrated.

\section{miR172 AFFECTS TUBERIZATION IN A GRAFT-TRANSMISSIBLE MANNER}

To date, miR172, which regulates flowering in several species, is the only microRNA (miRNA) shown to affect tuber induction (Martin etal., 2009; Zhu and Helliwell, 2011). The effect of miR172 in potato has been reported in overexpressing plants, which form tubers under LDs, tuberize early under SDs and show up-regulation of StBEL5. Inactivation would help to confirm if miR172 is required for tuberization control.

There is growing evidence that small RNAs, including short interfering RNAs (siRNAs) and miRNAs, move cell-to-cell and systemically (Himber etal., 2003; Yoo et al., 2004; Lin etal., 2008; Pant et al., 2008; Chitwood et al., 2009; Carlsbecker et al.,
2010; Dunoyer etal., 2010; Molnar etal., 2010). The effect of miR172 overexpression is graft transmissible, suggesting that this miRNA regulates long-distance signals that control tuberization or, alternatively, that miR172 itself is a mobile signal. In grafting experiments, miR172-overexpressing scions accelerated tuberization of WT stocks, but the reciprocal graft combination did not tuberize early. The simplest interpretation is that miR172 is required in aerial organs, rather than in stolons, to promote tuberization. However, increases of miR172 levels in stolons correlate with tuber induction, while changes in leaves do not (Martin et al., 2009). At least two hypotheses can explain this apparent contradiction: (1) overexpression of miR172 in stocks might not be sufficient to counteract tuber-inhibiting signals derived from WT 
scions; and (2) factors required for miR172 processing might be present or active in leaves but not in stolons. Detection of miR172 in potato phloem cells and phloem exudates of several species, as well as graft transmission in Nicotiana benthamiana, is consistent with the notion of this miRNA being mobile (Buhtz et al., 2008, 2010; Martin et al., 2009; Kasai et al., 2010; Varkonyi-Gasic et al., 2010). In addition to its putative role as a systemic signal, it has been proposed that miR172 might participate in cell-tocell communication (Abelenda et al., 2011; Marín-González and Suárez-López, 2012). Given the potential of miRNAs to act as transmissible signals, it will be worth studying whether miR172 moves.

\section{ROLE OF GIBBERELLINS IN TUBERIZATION}

The plant hormones gibberellins (GAs) are present in phloem sap and seem to act as florigenic molecules in some species (Eriksson et al., 2006; King et al., 2006, 2008). The last steps in the biosynthesis of active GAs are catalyzed by GA 20-oxidase (GA20ox) and GA 3-oxidase (GA3ox). Biologically active GAs, including $G_{1}$, $\mathrm{GA}_{3}$, and $\mathrm{GA}_{4}$, are inactivated by GA 2-oxidase (GA2ox) enzymes (Hedden and Thomas, 2012).

Gibberellins are involved in the control of tuber induction or development. Different observations have led to the assumption that GAs inhibit tuberization under LDs. Tuberization would take place when GA levels decrease in response to SDs (RodríguezFalcón etal., 2006). This decrease seems necessary to arrest longitudinal stolon growth and allow stolon swelling (Jackson, 1999). But are high GA levels really required to repress tuber induction under LDs? Silencing of a potato GA20ox (StGA20ox1) and manipulation of the levels of a GA3ox (StGA3ox2) do not induce tuberization under LDs (Carrera et al., 2000; Bou-Torrent et al., 2011). In addition, a GA2ox, StGA2ox1, affects tuberization in vitro, but not in soil-grown plants (Kloosterman et al., 2007), leading to the conclusion that StGA2ox1 is a tuber-identity gene rather than a regulator of tuber induction. Local up-regulation of StGA2ox1 in stolons by StSP6A (Navarro et al., 2011) is consistent with this interpretation.

Moreover, the expression patterns of several GA biosynthetic enzymes and the phenotypes of plants with altered levels of these enzymes do not always fit with the hypothesis of GAs repressing tuberization. For example, although $S t G A 30 \times 2$ is down-regulated at the initiation of tuber development, StGA20ox1 and StGA20ox3 are up-regulated (Kloosterman etal., 2007). Both StGA20ox1silenced lines and plants overexpressing StGA3ox2 tuberize earlier than WT plants under SDs, despite showing opposite changes of $\mathrm{GA}_{1}$ levels (Carrera et al., 2000; Bou-Torrent et al., 2011). As GA biosynthesis involves feedback and feedforward regulations (Hedden and Thomas, 2012), some of these contradictions can be explained through negative feedback regulation of StGA20ox genes by active GAs, but this still has to be demonstrated.

To explain some of these conflicting results, it has recently been proposed that $\mathrm{GA}_{20}$ - the immediate precursor of $\mathrm{GA}_{1}$ - would be mobile, whereas $\mathrm{GA}_{1}$ would not. In StGA3ox2-overexpressing plants, increased conversion of $\mathrm{GA}_{20}$ to $\mathrm{GA}_{1}$ in aerial parts would reduce the amount of $\mathrm{GA}_{20}$ transported to stolons, resulting in low levels of $\mathrm{GA}_{1}$ in stolons and early tuberization (Bou-Torrent et al., 2011). This interesting hypothesis fits well with some observations.
However, as StGA20ox genes are expressed in stolons (Carrera et al., 1999), GA 20 is expected to be synthesized here. StGA3ox2overexpressing plants would then have increased conversion of $\mathrm{GA}_{20}$ to $\mathrm{GA}_{1}$ also in stolons, which should repress tuberization. Localized silencing of StGA20ox1 and StGA3ox2 in leaves and stolons and grafting experiments using plants with altered levels of these enzymes would help to elucidate the role of $\mathrm{GA}_{20}$ and $\mathrm{GA}_{1}$. It will also be necessary to test $\mathrm{GA}_{20}$ movement in potato plants and whether movement is required to prevent tuberization. More work is also needed to determine whether GAs play a role in tuber induction or they regulate tuber development by preventing stolons from being competent to respond to leaf-derived inductive signals. Nowadays it cannot be excluded that GAs perform both functions.

\section{SUCROSE AND OTHER PUTATIVE LONG-RANGE SIGNALING MOLECULES}

Sucrose is a metabolite, a source of energy and a signaling molecule and it has been proposed as a transmissible substance for tuberization and flowering (Sheen et al., 1999; Suárez-López, 2005; Ruan, 2012). Transcripts of sucrose transporters are phloem mobile in several species, including potato, which suggests a possible signaling role for these RNAs (Liesche et al., 2011). A potato sucrose transporter, StSUT4, is involved in flowering and tuberization control. Inhibition of StSUT4 induces tuberization under LDs. Graft transmission of this phenotype, together with an increase in sucrose export from leaves of StSUT4-silenced plants, suggest a role for StSUT4 in long-distance signaling at least in part via source to sink carbon flux (Chincinska et al., 2008). In addition, StSUT4 regulates the production of putative long-distance signals, such as StSP6A and probably GAs (Chincinska et al., 2008, 2013).

There is additional evidence of a link between sucrose and GAs during tuberization. In vitro treatment with high sucrose concentrations, which induces tuber formation, reduces endogenous $\mathrm{GA}_{1}$ levels in stolons before tuber initiation (Xu et al., 1998). Exogenous GA treatment, conversely, up-regulates StSUT4 (Chincinska et al., 2008). Altogether these observations indicate a complex interplay between GAs and sucrose during tuber induction or development. Understanding the different roles that sucrose plays in tuber formation, as a starch precursor, energy source and signal, deserves further attention.

Other molecules, such as metabolites, hormones, and peptides have the potential to act as mobile signals, but their roles in tuberization are not yet clear (Jackson, 1999; Fernie and Willmitzer, 2001; Dinant and Suárez-López, 2012). Grafting of tomato mutants onto potato stocks has been proposed as a strategy to elucidate the role of hormones in long-distance signaling, although the results so far point to effects on assimilate distribution rather than on signaling pathways (Peres et al., 2005).

\section{THE ROLE OF PHYTOCHROME B IN REGULATING MOBILE SIGNALS}

The photoreceptor phytochrome B (PHYB) plays an interesting role in the control of tuber induction, as it affects several putative systemic tuberization molecules. Grafting experiments using PHYB-silenced plants, which tuberize under LDs, led to 
the proposal that PHYB induces a mobile tuberization repressor(Jackson et al., 1998). However, these plants show increased levels of StSP6A mRNA and reduced levels of StBEL5 mRNA and miR172 in leaves, as well as increased levels of these three RNAs in stolons (Martin et al., 2009; Navarro et al., 2011), indicating that PHYB inhibits the expression and/or movement of tuberinducing molecules. Probably positive and negative regulators of tuberization respond to light signals through the action of PHYB.

Several effects of PHYB on plant development are mediated by GAs (Lau and Deng, 2010). In potato, PHYB affects GA synthesis or signaling, as StGA20ox1 mRNA abundance is increased in PHYB-silenced plants, which show several phenotypes characteristic of alterations in GA homeostasis (Jackson et al., 2000). In addition, StSUT4 probably mediates some effects of PHYB on plant development (Chincinska et al., 2008). How PHYB regulates all these genes is not known. PHYB controls long-distance regulation of other processes, pointing to a general role of PHYB in systemic signaling (Griebel and Zeier, 2008; Suzuki et al., 2011).

\section{CONCLUSION AND FUTURE CHALLENGES}

We have recently witnessed substantial advances in our knowledge of potato tuber induction. Although the identity of mobile

\section{REFERENCES}

Abelenda, J. A., Navarro, C., and Prat, S. (2011). From the model to the crop: genes controlling tuber formation in potato. Curr. Opin. Biotecnol. 22, 287-292. doi: 10.1016/ j.copbio. 2010.11 .013

Aki, T., Shigyo, M., Nakano, R., Yoneyama, T., and Yanagisawa, S. (2008). Nano scale proteomics revealed the presence of regulatory proteins including three FT-Like proteins in phloem and xylem saps from rice. Plant Cell Physiol. 49, 767-790. doi: 10.1093/pcp/pcn049

An, H., Roussot, C., Suárez-López, P., Corbesier, L., Vincent, C., Piñeiro, M., et al. (2004). CONSTANS acts in the phloem to regulate a systemic signal that induces photoperiodic flowering of Arabidopsis. Development 131, 3615-3626. doi: 10.1242/dev.01231

Ayre, B. G., and Turgeon, R. (2004). Graft transmission of a floral stimulant derived from CONSTANS. Plant Physiol. 135, 2271-2278. doi: 10.1104/pp.104.040592

Banerjee, A. K., Chatterjee, M., Yu, Y., Suh, S.-G., Miller, W. A., and Hannapel, D. J. (2006). Dynamics of a mobile RNA of potato involved in a long-distance signaling pathway. Plant Cell 18, 3443-3457. doi: 10.1105/tpc.106.042473

Bernier, G. (1988). The control of floral evocation and morphogenesis. Annu. Rev. Plant Physiol. Plant Mol. Biol. 39, 175-219. doi: 10.1146/annurev.pp.39.060188. 001135
Bou-Torrent, J., Martínez-García, J. F., (2011). Gibberellin A1 metabolism photoperiod-mediated tuberization in potato. PLoS ONE 6:e24458. doi: 10.1371/journal.pone.0024458

Buhtz, A., Pieritz, J., Springer, F. and Kehr, J. (2010). Phloem small RNAs, nutrient stress responses, and systemic mobility. BMC Plant Biol. 10:64. doi: 10.1186/1471-2229-10-64

Buhtz, A., Springer, F., Chappell, L., Baulcombe, D. C., and Kehr, J. (2008). Identification and characterization of small RNAs from the phloem of Brassica napus. Plant J. 53, 739749. doi: 10.1111/j.1365-313X.2007. 03368.x

Carlsbecker, A., Lee, J.-Y., Roberts, C. J., Dettmer, J., Lehesranta, S., Zhou, J., etal. (2010). Cell signalling by microRNA165/6 directs gene dosedependent root cell fate. Nature 465, 316-321. doi: 10.1038/nature08977

Carrera, E., Bou, J., García-Martínez, J. L., and Prat, S. (2000). Changes in GA 20-oxidase gene expression strongly affect stem length, tuber induction and tuber yield of potato plants. Plant J. 22, 247-256. doi: 10.1046/j.1365313x.2000.00736.x

Carrera, E., Jackson, S. D., and Prat, S. (1999). Feedback control and diurnal regulation of gibberellin 20oxidase transcript levels in potato. Plant Physiol. 119, 765-773. doi: 10.1104/pp.119.2.765

Chailakhyan, M. K., Yanina, L. I., Devedzhyan, A. G., and Lotova, G. García-Martínez, J. L., and Prat, S. contributes to the control of

tuberization molecules is yet unknown, they are probably similar to flowering signals. Several good candidates have been proposed (Figure 1). Further research should test whether they act as genuine systemic tuberization signals.

Long-distance communication involves the production of signals, but also requires phloem loading, transport and unloading, as well as the response of target tissues to the translocated signals. Once the chemical nature of the signals is established, dissecting all these processes will be easier. The availability of the potato genome sequence (Xu et al., 2011) will facilitate these tasks. Interspecific grafting and experimental approaches used in other species, such as analyses of phloem sap composition, visualization of reporters fused to putatively mobile proteins and strategies to disrupt intercellular signaling, can be employed to address these questions.

\section{ACKNOWLEDGMENTS}

I acknowledge funding by Spanish MINECO and MICINN (Grants BFU2012-33746 and CSD2007-00036 "Centre for Research in Agrigenomics") and the Department of Innovation, Universities and Enterprise of Catalonia (2009-SGR-703 and Catalan Biotechnology Reference Network).

N. (1981). Photoperiodism and tuber formation in grafting of tobacco onto potato. Dokl. Akad. Nauk 257, 1276 1280.

Chapman, H. W. (1958). Tuberization in the potato plant. Physiol. Plant. 11, 215-224. doi: 10.1111/j.13993054.1958.tb08460.x

Chen, H., Rosin, F. M., Prat, S. and Hannapel, D. J. (2003). Interacting transcription factors from the three-amino acid loop extension superclass regulate tuber formation. Plant Physiol. 132, 1391-1404. doi: 10.1104/pp.103.022434

Chincinska, I., Gier, K., Krügel, U., Liesche, J., He, H., Grimm, B., et al. (2013). Photoperiodic regulation of the sucrose transporter StSUT4 affects the expression of circadian-regulated genes and ethylene production. Front. Plant Sci. 4:26. doi: 10.3389/fpls.2013.00026

Chincinska, I. A., Liesche, J., Krügel, U., Michalska, J., Geigenberger, P. Grimm, B., et al. (2008). Sucrose transporter StSUT4 from potato affects flowering, tuberization, and shade avoidance response. Plant Physiol. 146, 515-528. doi: 10.1104/ pp.107.112334

Chitwood, D. H., Nogueira, F. T., Howell, M. D., Montgomery, T. A., Carrington, J. C., and Timmermans, M. C. (2009). Pattern formation via small RNA mobility. Genes Dev. 23, 549-554. doi: 10.1101/gad.1770009

D’Aloia, M., Bonhomme, D., Bouché, F., Tamseddak, K., Ormenese, S., Torti, S., et al. (2011). Cytokinin promotes flowering of Arabidopsis via transcriptional activation of the FT paralogue TSF. Plant J. 65, 972-979. doi: 10.1111/j.1365-313X.2011.04482.x

Dinant, S., and Suárez-López, P. (2012). "Multitude of long-distance signal molecules via phloem," in Biocommunication of Plants, eds G. Witzany and F. Baluška (Heidelberg: Springer), 89-121. doi: 10.1077/9783-642-23524-5_6

Doering-Saad, C., Newbury, H. J., Bale, J. S., and Pritchard, J. (2002). Use of aphid stylectomy and RT-PCR for the detection of transporter mRNAs in sieve elements. J. Exp. Bot. 53, 631637. doi: 10.1093/jexbot/53.369.631

Dunoyer, P., Schott, G., Himber, C., Meyer, D., Takeda, A., Carrington, J. C., et al. (2010). Small RNA duplexes function as mobile silencing signals between plant cells. Science 328, 912916. doi: 10.1126/science. 1185880

Eriksson, S., Böhlenius, H., Moritz, T., and Nilsson, O. (2006). $\mathrm{GA}_{4}$ is the active gibberellin in the regulation of LEAFY transcription and Arabidopsis floral initiation. Plant Cell 18, 2172-2181. doi: 10.1105/tpc.106. 042317

Ewing, E. E., and Struik, P. C. (1992). Tuber formation in potato: induction, initiation and growth. Hortic. Rev. 14, 89-198. doi: 10.1002/ 9780470650523.ch3

Fernie, A. R., and Willmitzer, L. (2001). Molecular and biochemical triggers of potato tuber development. Plant Physiol. 127, 1459-1465. doi: 10.1104/pp.010764 
Giavalisco, P., Kapitza, K., Kolasa, A., Buhtz, A., and Kehr, J. (2006). Towards the proteome of Brassica napus phloem sap. Proteomics 6, 896909. doi: 10.1002/pmic.200500155

González-Schain, N. D., Díaz-Mendoza, M., Żurczak, M., and Suárez-López, P. (2012). Potato CONSTANS is involved in photoperiodic tuberization in a graft-transmissible manner. Plant J. 70, 678-690. doi: 10.1111/j.1365-313X.2012.04909.x

Gregory, L. E. (1956). Some factors for tuberization in the potato plant. Am. J. Bot. 43, 281-288. doi: $10.2307 / 2438945$

Griebel, T., and Zeier, J. (2008). Light regulation and daytime dependency of inducible plant defenses in Arabidopsis: phytochrome signaling controls systemic acquired resistance rather than local defense. Plant Physiol. 147, 790-801. doi: 10.1104/pp.108.119503

Guo, J.-L., Yu, C.-L., Fan, C.-Y., Lu, Q.-N., Yin, J.-M., Zhang, Y.-F., et al. (2010). Cloning and characterization of a potato TFL1 gene involved in tuberization regulation. Plant Cell Tissue Organ Cult. 103, 103-109. doi: 10.1007/s11240-010-9759-8

Hannapel, D. J. (2010). A model system of development regulated by the long-distance transport of mRNA. J. Integr. Plant Biol. 52, 40-52. doi: 10.1111/j.1744-7909.2010.00911.x

Haywood, V., Yu, T.-S., Huang, N.-C., and Lucas, W. J. (2005). Phloem longdistance trafficking of GIBBERELLIC ACID-INSENSITIVE RNA regulates leaf development. Plant J. 42, 4968. doi: 10.1111/j.1365-313X.2005. 02351.x

Hedden, P., and Thomas, S. G. (2012). Gibberellin biosynthesis and its regulation. Biochem. J. 444, 11-25. doi: 10.1042/BJ20120245

Himber, C., Dunoyer, P., Moissiard, G., Ritzenthaler, C., and Voinnet, O. (2003). Transitivity-dependent and -independent cell-to-cell movement of RNA silencing. EMBO J. 22, 4523-4533. doi: 10.1093/emboj/ cdg431

Hiraoka, K., Yamaguchi, A., Abe, M., and Araki, T. (2013). The florigen genes FT and TSF modulate lateral shoot outgrowth in Arabidopsis thaliana. Plant Cell Physiol. 54, 352368. doi: $10.1093 / \mathrm{pcp} / \mathrm{pcs} 168$

Huang, N.-C., Jane, W.-N., Chen, J., and Yu, T.-S. (2012). Arabidopsis thaliana CENTRORADIALIS homologue (ATC) acts systemically to inhibit floral initiation in Arabidopsis. Plant J. 72, 175184. doi: 10.1111/j.1365-313X.2012. 05076.x
Jackson, S. D. (1999). Multiple signaling pathways control tuber induction in potato. Plant Physiol. 119, 1-8. doi: 10.1104/pp.119.1.1

Jackson, S. D., James, P., Prat, S., and Thomas, B. (1998). Phytochrome B affects the levels of a graft-transmissible signal involved in tuberization. Plant Physiol. 117, 2932. doi: 10.1104/pp.117.1.29

Jackson, S. D., James, P. E., Carrera, E., Prat, S., and Thomas, B. (2000). Regulation of transcript levels of a potato gibberellin 20-oxidase gene by light and phytochrome $\mathrm{B}$. Plant Physiol. 124, 423-430. doi: 10.1104/pp.124.1.423

Jang, S., Torti, S., and Coupland, G. (2009). Genetic and spatial interactions between FT, TSF and SVP during the early stages of floral induction in Arabidopsis. Plant J. 60, 614-625. doi: 10.1111/j.1365313X.2009.03986.x

Kasai, A., Kanehira, A., and Harada, T. (2010). miR172 can move long distances in Nicotiana benthamiana. Open Plant Sci. J. 4, 1-6. doi: 10.2174/1874294701004010001

Kim, M., Canio, W., Kessler, S. and Sinha, N. (2001). Developmental changes due to longdistance movement of a homeobox fusion transcript in tomato. Science 293, 287-289. doi: 10.1126/science. 1059805

King, R. W., Mander, L. N., Asp, T., MacMillan, C. P., Blundell, C. A., and Evans, L. T. (2008). Selective deactivation of gibberellins below the shoot apex is critical to flowering but not to stem elongation of Lolium. Mol. Plant 1, 295-307. doi: 10.1093/mp/ ssm030

King, R. W., Moritz, T., Evans, L. T., Martin, J., Andersen, C. H., Blundell, C., et al. (2006). Regulation of flowering in the longday grass Lolium temulentum by gibberellins and the FLOWERING LOCUS $T$ gene. Plant Physiol. 141, 498-507. doi: 10.1104/pp.106. 076760

Kloosterman, B., Abelenda, J. A., Gomez, M. d. M. C., Oortwijn, M., de Boer, J. M., Kowitwanich, K., et al. (2013). Naturally occurring allele diversity allows potato cultivation in northern latitudes. Nature 495, 246250. doi: 10.1038/nature11912

Kloosterman, B., Navarro, C., Bijsterbosch, G., Lange, T., Prat, S., Visser, R. G. F., et al. (2007). StGA2oxl is induced prior to stolon swelling and controls GA levels during potato tuber development. Plant J. 52, 362-373. doi: $10.1111 / \mathrm{j} .1365$ 313X.2007.03245.x
Komiya, R., Yokoi, S., and Shimamoto K. (2009). A gene network for longday flowering activates RFT1 encoding a mobile flowering signal in rice. Development 136, 3443-3450. doi: 10.1242/dev.040170

Lau, O. S., and Deng, X. W. (2010). Plant hormone signaling lightens up: integrators of light and hormones. Curr. Opin. Plant Biol. 13, 571-577. doi: 10.1016/j.pbi.2010.07.001

Li, C., Gu, M., Shi, N., Zhang, H. Yang, X., Osman, T., et al. (2011). Mobile FT mRNA contributes to the systemic florigen signalling in floral induction. Sci. Rep. 1, 73. doi: 10.1038/srep00073

Li, C., Zhang, K., Zeng, X., Jackson, S., Zhou, Y., and Hong, Y. (2009). A cis element within Flowering Locus $T$ mRNA determines its mobility and facilitates trafficking of heterologous viral RNA. J. Virol. 83, 3540-3548. doi: 10.1128/JVI.02346-08

Liesche, J., Krügel, U., He, H., Chincinska, I., Hackel, A., and Kühn, C. (2011). Sucrose transporter regulation at the transcriptional, post-transcriptional and posttranslational level. J. Plant Physiol. 168, 1426-1433. doi: 10.1016/j.jplph. 2011.02.005

Lifschitz, E., Eviatar, T., Rozman, A., Shalit, A., Goldshmidt, A., Amsellem, Z., et al. (2006). The tomato FT ortholog triggers systemic signals that regulate growth and flowering and substitute for diverse environmental stimuli. Proc. Natl. Acad. Sci. U.S.A. 103, 6398-6403. doi: 10.1073/pnas.0601620103

Lin, M.-K., Belanger, H., Lee, Y. J., Varkonyi-Gasic, E., Taoka, K.-I., Miura, E., et al. (2007). FLOWERING LOCUS T protein may act as the long-distance florigenic signal in the cucurbits. Plant Cell 19, 1488-1506. doi: 10.1105/tpc.107.051920

Lin, S.-I., Chiang, S.-F., Lin, W.-Y., Chen, J.-W., Tseng, C.-Y., Wu, P. C., et al. (2008). Regulatory network of microRNA399 and PHO2 by systemic signaling. Plant Physiol. 147, 732-746. doi: 10.1104/pp.108. 116269

Lu, K.-J., Huang, N.-C., Liu, Y.-S., Lu, C.-A., and Yu, T.-S. (2012). Longdistance movement of Arabidopsis FLOWERING LOCUS T RNA participates in systemic floral regulation. RNA Biol. 9, 653-662. doi: 10.4161/rna.19965

Mahajan, A., Bhogale, S., Kang, I., Hannapel, D., and Banerjee, A. (2012). The mRNA of a Knotted1-like transcription factor of potato is phloem mobile. Plant Mol. Biol. 79, 595-608. doi: $10.1007 /$ s11103-012-9931-0
Marín-González, E., and Suárez-López, P. (2012). "And yet it moves": cell-to-cell and long-distance signaling by plant microRNAs. Plant Sci. 196, 18-30. doi: 10.1016/ j.plantsci.2012.07.009

Martin, A., Adam, H., Díaz-Mendoza, M., Żurczak, M., GonzálezSchain, N. D., and Suárez-López, P. (2009). Graft-transmissible induction of potato tuberization by the microRNA miR172. Development 136, 2873-2881. doi: 10.1242/ dev.031658

Mathieu, J., Warthmann, N., Küttner, F., and Schmid, M. (2007). Export of FT protein from phloem companion cells is sufficient for floral induction in Arabidopsis. Curr. Biol. 17, 10551060. doi: 10.1016/j.cub.2007.05.009

Matsoukas, I. G., Massiah, A. J., and Thomas, B. (2012). Florigenic and antiflorigenic signalling in plants. Plant Cell Physiol. 53, 1827-1842. doi: 10.1093/pcp/pcs130

Molnar, A., Melnyk, C. W., Bassett, A., Hardcastle, T. J., Dunn, R., and Baulcombe, D. C. (2010). Small silencing RNAs in plants are mobile and direct epigenetic modification in recipient cells. Science 328, 872-875. doi: 10.1126/science.1187959

Navarro, C., Abelenda, J. A., CruzOró, E., Cuéllar, C. A., Tamaki, S., Silva, J., et al. (2011). Control of flowering and storage organ formation in potato by FLOWERING LOCUS T. Nature 478, 119-122. doi: 10.1038/nature 10431

Notaguchi, M., Abe, M., Kimura, T., Daimon, Y., Kobayashi, T., Yamaguchi, A., etal. (2008). Longdistance, graft-transmissible action of Arabidopsis FLOWERING LOCUS T protein to promote flowering. Plant Cell Physiol. 49, 1645-1658. doi: 10.1093/pcp/pcn 154

Pant, B. D., Buhtz, A., Kehr, J., and Scheible, W.-R. (2008). MicroRNA399 is a long-distance signal for the regulation of plant phosphate homeostasis. Plant J. 53, 731-738. doi: 10.1111/j.1365313X.2007.03363.x

Peres, L. E. P., Carvalho, R. F., Zsögön, A., Bermúdez-Zambrano, O. D., Robles, W. G. R., and Tavares, S. (2005). Grafting of tomato mutants onto potato rootstocks: an approach to study leaf-derived signaling on tuberization. Plant Sci. 169, 680-688. doi: 10.1016/j.plantsci.2005.05.017

Pin, P. A., and Nilsson, O. (2012). The multifaceted roles of FLOWERING LOCUS $\mathrm{T}$ in plant development. Plant Cell Environ. 35, 1742-1755. doi: 10.1111/j.13653040.2012.02558.x 
Rodríguez-Falcón, M., Bou, J., and Prat, S. (2006). Seasonal control of tuberization in potato: conserved elements with the flowering response. Annu. Rev. Plant Biol. 57, 151-180. doi: 10.1146/annurev.arplant.57.032905. 105224

Rosin, F. M., Hart, J. K., Horner, H. T., Davies, P. J., and Hannapel, D. J. (2003). Overexpression of a Knotted-like homeobox gene of potato alters vegetative development by decreasing gibberellin accumulation. Plant Physiol. 132, 106-117. doi: 10.1104/pp.102.015560

Ruan, Y.-L. (2012). Signaling role of sucrose metabolism in development. Mol. Plant 5, 763-765. doi: 10.1093/mp/sss046

Ruiz-Medrano, R., Xoconostle-Cázares, B., and Lucas, W. J. (1999). Phloem long-distance transport of CmNACP mRNA: implications for supracellular regulation in plants. Development 126, 44054419.

Sasaki, T., Chino, M., Hayashi, H., and Fujiwara, T. (1998). Detection of several mRNA species in rice phloem sap. Plant Cell Physiol. 39, 895-897. doi: 10.1093/oxfordjournals.pcp.a029451

Sheen, J., Zhou, L., and Jang, J.-C. (1999). Sugars as signaling molecules. Curr. Opin. Plant Biol. 2,
410-418. doi: 10.1016/S1369-5266 (99)00014-X

Suárez-López, P. (2005). Long-range signalling in plant reproductive development. Int. J. Dev. Biol. 49, 761-771. doi: 10.1387/ijdb.052002ps Suzuki, A., Suriyagoda, L., Shigeyama, T., Tominaga, A., Sasaki, M., Hiratsuka, Y., et al. (2011). Lotus japonicus nodulation is photomorphogenetically controlled by sensing the red/far red $(\mathrm{R} / \mathrm{FR})$ ratio through jasmonic acid (JA) signaling. Proc. Natl. Acad. Sci. U.S.A. 108, 16837-16842. doi: 10.1073/pnas.1105892108

Tamaki, S., Matsuo, S., Wong, H. L., Yokoi, S., and Shimamoto, K. (2007). $\mathrm{Hd} 3$ a protein is a mobile flowering signal in rice. Science 316, 1033-1036. doi: 10.1126/science.1141753

Tsuji, H., Taoka, K.-I., and Shimamoto, K. (2013). Florigen in rice: complex gene network for florigen transcription, florigen activation complex, and multiple functions. Curr. Opin. Plant Biol. 16, 228-35. doi: 10.1016/j.pbi.2013.01.005 doi: 10.1016/j.pbi.2013.01.005

Turck, F., Fornara, F., and Coupland, G. (2008). Regulation and identity of florigen: FLOWERING LOCUS T moves center stage. Annu. Rev. Plant Biol. 59, 573-594. doi: 10.1146/annurev.arplant.59.032607. 092755
Turnbull, C. (2011). Long-distance regulation of flowering time. J. Exp. Bot. 62, 4399-4413. doi: 10.1093/jxb/ err191

Varkonyi-Gasic, E., Gould, N., Sandanayaka, M., Sutherland, P., and MacDiarmid, R. (2010). Characterisation of microRNAs from apple (Malus domestica 'Royal Gala') vascular tissue and phloem sap. BMC Plant Biol. 10:159. doi: 10.1186/1471-222910-159

Xu, X., Pan, S., Cheng, S., Zhang, B. $\mathrm{Mu}, \mathrm{D}$., Ni, P., et al. (2011). Genome sequence and analysis of the tuber crop potato. Nature 475, 189-195. doi: $10.1038 /$ nature 10158

Xu, X., van Lammeren, A. A. M., Vermeer, E., and Vreugdenhil, D. (1998). The role of gibberellin, abscisic acid, and sucrose in the regulation of potato tuber formation in vitro. Plant Physiol. 117, 575-584. doi: 10.1104/pp.117.2.575

Yamaguchi, A., Kobayashi, Y., Goto, K., Abe, M., and Araki, T. (2005). TWIN SISTER OF FT (TSF) acts as a floral pathway integrator redundantly with FT. Plant Cell Physiol. 46, 1175-1189. doi: 10.1093/pcp/ pci151

Yoo, B.-C., Kragler, F., Varkonyi-Gasic, E., Haywood, V., Archer-Evans, S., Lee, Y. M., et al. (2004). A systemic small RNA signaling system in plants. Plant Cell 16, 1979-2000. doi: 10.1105/tpc.104.023614

Zhu, Q.-H., and Helliwell, C. A. (2011). Regulation of flowering time and floral patterning by miR172. J. Exp. Bot. 62, 487-495. doi: 10.1093/jxb/ erq295

Conflict of Interest Statement: The author declares that the research was conducted in the absence of any commercial or financial relationships that could be construed as a potential conflict of interest.

Received: 02 April 2013; paper pending published: 19 April 2013; accepted: 23 June 2013; published online: 16 July 2013.

Citation: Suárez-López P (2013) A critical appraisal of phloem-mobile signals involved in tuber induction. Front. Plant Sci. 4:253. doi: 10.3389/fpls.2013.00253 This article was submitted to Frontiers in Plant Physiology, a specialty of Frontiers in Plant Science.

Copyright () 2013 Suárez-López. This is an open-access article distributed under the terms of the Creative Commons Attribution License, which permits use, distribution and reproduction in other forums, provided the original authors and source are credited and subject to any copyright notices concerning any thirdparty graphics etc. 